\title{
Wind Tunnel to Atmospheric Mapping for Static Aeroelastic Scaling
}

\author{
Jennifer Heeg ${ }^{\text {}}$, Charles V. Spain ${ }^{\star}$, J.A. Rivera ${ }^{\dagger}$ \\ NASA Langley Research Center, Hampton VA 23681.
}

\begin{abstract}
Wind tunnel to Atmospheric Mapping (WAM) is a methodology for scaling and testing a static aeroelastic wind tunnel model. The WAM procedure employs scaling laws to define a wind tunnel model and wind tunnel test points such that the static aeroelastic flight test data and wind tunnel data will be correlated throughout the test envelopes. This methodology extends the notion that a single test condition- combination of Mach number and dynamic pressure- can be matched by wind tunnel data. The primary requirements for affecting this extension are matching flight Mach numbers, maintaining a constant dynamic pressure scale factor and setting the dynamic pressure scale factor in accordance with the stiffness scale factor. The scaling is enabled by capabilities of the NASA Langley Transonic Dynamics Tunnel (TDT) and by relaxation of scaling requirements present in the dynamic problem that are not critical to the static aeroelastic problem. The methodology is exercised in two example scaling problems: an arbitrarily scaled wing and a practical application to the scaling of the Active Aeroelastic Wing flight vehicle for testing in the TDT.
\end{abstract}

\section{Introduction}

Experimental research in aeroelasticity is often thought of as flutter testing. Because of safety, flutter clearance is considered critical in developing new aircraft systems and typically involves the development and testing of dynamic aeroelastically-scaled wind tunnel models prior to full-scale prototype development. Experimental static aeroelasticity however, is often only considered after prototype development, and typically involves flight testing of the full-scale aircraft. Modern aircraft however, may benefit from an increased emphasis on wind-tunnel static aeroelastic experimentation. For example, experimentbased flexible stability and control derivatives would be more reliable than analysis-based, and would be available to designers prior to prototype development. This paper describes how the classic aeroelastic scaling laws can be adapted for static aeroelasticity, thereby making static aeroelastic wind-tunnel model development somewhat easier and more practical.

Wind tunnel to Atmospheric Mapping (WAM) is a methodology for scaling and testing a static aeroelastic wind tunnel model. The WAM procedure employs scaling laws to define a wind tunnel model and wind tunnel test points such that the static aeroelastic flight test data and wind tunnel data correlate throughout the test envelopes. This

${ }^{D}$ Senior Research Engineer, Aeroelasticity Branch, Mail Stop 340, Senior Member AIAA

* Senior Research Engineer, Aeroelasticity Branch, Mail Stop 340

${ }^{\dagger}$ Senior Aerospace Engineer, Aeroelasticity Branch, Mail Stop 340 methodology extends the notion that a single test condition -combination of Mach number and dynamic pressure- can be matched by wind tunnel data. The scaling is enabled by capabilities of the NASA Langley Transonic Dynamics Tunnel (TDT) and by relaxation of scaling requirements present in the dynamic problem that are not critical to the static aeroelastic problem.

Scaling a model such that it is staticallyaeroelastically similar to an airplane requires that its characteristics under steady loads match those of the airplane. ${ }^{1}$ A model that is statically-aeroelastically scaled to a flight vehicle deflects to the same shape and with scaled magnitude under scaled static loads.

This paper explains the procedure used to develop scale factors for the statically-aeroelastically scaled wind tunnel model and the requirements for acquiring and scaling the resultant data. Pertinent assumptions, restrictions, limitations and implications of this methodology are also discussed. Two analytical examples of applying the WAM methodology are presented: a hypothetical arbitrarily scaled wing and the Active Aeroelastic Wing.

Experimental implementation of this methodology is expected to utilize unique features of the NASA Langley Transonic Dynamics Tunnel (TDT). Thus, pertinent capabilities and systems of this facility are examined. Practical details and the effects of errors are examined. Resolution limitations of wind tunnel instrumentation and uncertainties in calculated wind tunnel flow parameters are mapped to flight test conditions, providing limits for the precision with which flight test conditions can be matched. 


\section{Nomenclature}

$\begin{array}{ll}\mathrm{a} & \text { speed of sound } \\ \mathrm{b} & \text { semi-span } \\ \mathrm{E} & \text { Young's modulus } \\ \mathrm{Fr} & \text { Froude number } \\ \mathrm{g} & \text { gravitational acceleration } \\ \mathrm{h} & \text { altitude } \\ \mathrm{I} & \text { inertia } \\ \mathrm{L} & \text { length } \\ \mathrm{M} & \text { Mach number } \\ M & \text { moment applied } \\ p & \text { distributed load } \\ \mathrm{P} & \text { force applied } \\ \overline{\mathbf{q}} & \text { dynamic pressure } \\ \mathrm{S} & \text { wing area } \\ \mathrm{V} & \text { velocity } \\ \mathrm{x} & \text { spanwise coordinate } \\ \mathrm{y} & \text { out-of-plane deflection } \\ \rho & \text { density }\end{array}$

\section{Scale Factors}

$\lambda$ (variable) ..... scale factor on (variable) $=(\text { variable })_{\mathrm{M}} /(\text { variable })_{\mathrm{A}}$

(example: $\quad \lambda_{\mathrm{L}}=\mathrm{L}_{\mathrm{M}} / \mathrm{L}_{\mathrm{A}}=$ scale factor on length)

Subscripts \& Superscripts

A ...... pertaining to the aircraft

M ...... pertaining to wind tunnel model

' ...... non-dimensionalized quantity

o ...... reference quantity

\section{Background}

The Active Aeroelastic Wing Program

The Active Aeroelastic Wing (AAW) program is a cooperative effort among NASA, the Air Force Research Lab and Boeing Aircraft Corporation. The program objective is to develop technologies that will allow for the favorable use of aeroelastic properties of a flexible wing aircraft. The program consists of flight testing, wind tunnel testing and analyses. The primary purpose of the wind tunnel activities is to provide a testbed that is statically aeroelastically scaled to the flight test vehicle. A three-way data correlation involving flight test data, wind tunnel test data and analytical predictions will be performed. Open loop flight data has already been obtained; wind tunnel testing is scheduled to be conducted in the NASA Langley Transonic Dynamics Tunnel (TDT) late this year. This reversed schedule provides an opportunity to precisely match test conditions at which the flight test data has been acquired by carefully selecting and setting the wind tunnel test conditions.

\section{The Transonic Dynamics Tunnel}

The TDT is a closed circuit reduced pressure tunnel located at sea level. There are two choices of test medium: air and R134a heavy gas. The speeds of sound for the test media at atmospheric sea level pressure are approximately $1115 \mathrm{ft} / \mathrm{sec}$ for air and $540 \mathrm{ft} / \mathrm{sec}$ for R134a. The higher density and lower speed of sound associated with the heavy gas enable more latitude in model construction. The variable pressure allows for independent control over Mach number and dynamic pressure.

The TDT data system utilizes measurements of total pressure, static pressure, heavy gas purity and total temperature to compute additional flow parameters such as Mach number, dynamic pressure, density, velocity and speed of sound. Calculations are performed essentially in real time for isentropic flow properties of a real gas, both thermally and calorically imperfect, valid for arbitrary mixtures of R-134a and air. ${ }^{1,2}$

The tunnel operates most efficiently by testing along so-called wind-off total pressure lines, shown in figure 1. These operational lines are the Mach number-dynamic pressure combinations generated by establishing a wind-off total pressure and increasing Mach and dynamic pressure by changing motor RPM. The operational lines differ from lines of constant total pressure because the tunnel has inefficiencies and is not a truly "closed system." Energy exchanges between the thermodynamic system involved in the flow calculations and the surroundings are primarily due to energy input by the motor, and energy removal through cooling water and frictional heating. The energy losses experienced in operating a wind tunnel will vary from one facility to another, affecting a tunnel's ability to obtain data required for implementing the current scaling process. The energy losses in the TDT are sufficiently small to allow efficient testing at conditions required to match constant altitude flight data.

\section{Theory}

Aeroelastic similitude between a flight vehicle and a wind tunnel model can be achieved by matching non-dimensional parameters that govern the aerodynamics, the structure and their coupling. This problem was addressed in depth by W.G.Molyneux in $1964^{1}$. In this work, he presents the governing non-dimensional differential equations and discusses simplifications appropriate to different flow regimes and for different aspects of wind tunnel testing. Much of the theory discussed here is credited to Molyneux- the reader is encouraged to consult reference 1 when extending these concepts to other applications.

Aeroelastic similitude produces a long list of required similarity parameters and constraints. For similitude of the aerodynamics, the Mach number and Reynolds number must match and the bodies must be geometrically similar at the surface. This 
implies that not only must the shapes of the different bodies be the same, but also there must be similarity in their incidences to the flow and in their static elastic deformations. From the structural force-deflection equations, similitude requires that the ratio of stiffness to aerodynamic forces be maintained as well as the distribution of the stiffness throughout the structure. The vibration equations require mass ratio and stiffness ratio (or reduced frequency) to be maintained. The mass ratio is a ratio of vehicle mass to mass of the surrounding airspace; the reduced frequency represents the ratio of speed associated with the oscillations to the forward speed of the vehicle. The equilibrium equations require that mass ratio and Froude number be maintained. ${ }^{1,4,5,6}$

\section{Simplifications}

Design, construction and testing of a static aeroelastic wind tunnel model allows neglecting mass ratio, Reynolds number and Froude number under certain circumstances.

The mass ratio influences the aeroelastic problem if the dynamics are considered. For studying static aeroelastic phenomena, the requirements pertinent to dynamic characteristics are not necessary and can be neglected. This is a fundamental difference from the required methodology for producing a scaled flutter model.

The aerodynamic equations specify that the airfoil shape must be preserved, along with the Reynolds number and Mach number. The Reynolds number dictates the flow behavior in the boundary layer. Quoting Molyneux ${ }^{1}$, "For model tests to represent full scale conditions at reasonable air density (altitude) conditions, a compressed air tunnel is required. The difficulty arising from an excessive density scale derives from the necessity to satisfy the Reynolds number parameter. The Reynolds number is of paramount importance in relation to effects in the boundary layer. Experience has shown that it is generally of minor importance as far as aeroelastic effects for main lifting surfaces are concerned. If the Reynolds number requirement is ignored, the density scale becomes a free parameter, i.e., it is independent of the linear scale."

The Reynolds number effects are neglected in the current design and testing procedure. With the TDT capabilities, it is usually impossible to match the flight test Reynolds number. If it could be matched, meeting this criterion provides another constraint, limiting the free parameter selection. In neglecting the Reynolds number, the inherent assumption is that the boundary layer effects are not significant here. In the case of control surface aerodynamics, or flow separation phenomena, this may be a poor assumption ${ }^{1,4}$. In order to circumvent concerns regarding boundary layer transition, aeroelastic testing often requires tripping the boundary layer using grit applied near the leading edge of the model.

There are several types of static aeroelastic model programs where the velocity ratio, or Froude number, can be important. The Froude number determines the ratio of the deflections under steady gravitational load to deflections due to aerodynamic and inertial loads. For a Froude-scaled model, the aerodynamic and elastic forces are in proportion to the gravitational forces. In the case of a free-flying model, Froude scaling is important because gravity is providing one of the loads to achieve equilibrium. Neglecting Froude number would lead to the model lift in the equilibrium condition being disproportionate relative to that of the full-scale vehicle. In addition to the overall rigid body loads being maintained, the load distribution for an aeroelastic wing is important. In most cases, the deflection due to gravitational loading for a cantilever model will be insignificant compared to the aerodynamic loading. Froude scaling may be important, however, for low-speed aeroelastic models ${ }^{4}$. The gravitational contribution to the loading is important near zero velocity, but is quickly overwhelmed as the dynamic pressure increases. This is true in the case of either a free-flying or a supported model. For a free-to-roll model, Froude number might also be important because the gravitational forces may be significant when considering out-of-plane motion. Finally, in some cases, the wing may twist enough to significantly alter the center of gravity location and thus the gravitational force distribution; this may be critical in rolling power investigations ${ }^{1}$.

In the case of a cantilevered model, the supporting wall provides the forces and moments needed for equilibrium and restricts the model from rolling. Thus, Froude number matching is not required.

\section{Force-Deflection Relationship}

Understanding the requirements that arise from the force-deflection relationship is essential to understanding static aeroelastic scaling methodology. The general equations are given in reference 1; a simple example of a force-deflection relationship will be given here. While these equations do not represent realistic aerodynamic loading of an aircraft, the presented example evidences the scaling relationship between the magnitude of the load and the structural response.

Consider a uniform cantilevered beam under uniform distributed load of strength $p$, force per unit length. The beam bending equation is shown, eqn 1 .

$$
E I \frac{d^{2} y}{d x^{2}}=M(x)
$$


Integrating twice and applying boundary conditions of no deflection or slope at the cantilevered end, the deflection of the beam is given by eqn 2 .

$$
y=\frac{p}{E I}\left(-1 / 24 x^{4}+1 / 6 b x^{3}\right)
$$

The distribution of the force is buried in the integration. While this example utilizes a uniform force distribution, a realistic aerodynamic or aeroelastic problem would utilize a Mach number-dependent pressure distribution.

The following non-dimensional quantities are introduced and denoted by '. Notice that the force, $\mathrm{P}$, is non-dimensionalized in the same manner that an aerodynamic force is converted into a non-dimensional coefficient.

$$
\begin{array}{ll}
y^{\prime}=\frac{y}{L} ; \quad x^{\prime}=\frac{x}{L} ; \quad b^{\prime}=\frac{b}{L} \quad S^{\prime}=\frac{S}{L^{2}} ; \quad E^{\prime}=\frac{E}{E_{0}} ; \\
I^{\prime}=\frac{I}{I_{0}} ; \quad P^{\prime}=\frac{P}{\bar{q} S} ; \quad p^{\prime}=\frac{P^{\prime}}{b^{\prime}}=\frac{P L}{\bar{q} S b}=\frac{1}{\bar{q} S^{\prime} L} p
\end{array}
$$

Substituting the non-dimensional variables into the governing force-deflection eqn, 2 , yields

$$
\boldsymbol{y}^{\prime} \boldsymbol{L}=\frac{p^{\prime} \bar{q} S^{\prime} L}{E_{0} E^{\prime} I_{0} I^{\prime}}\left(-1 / 24 x^{\prime 4} L^{4}+1 / 6 b^{\prime} x^{\prime 3} L^{4}\right) \quad \text { Eqn } 3
$$

Rearranging,

$$
y^{\prime}=\left(\frac{\bar{q} L^{4}}{E_{0} I_{0}}\right) \frac{p^{\prime} S^{\prime}}{E^{\prime} I^{\prime}}\left(-1 / 24 x^{\prime 4}+1 / 6 b^{\prime} x^{3}\right) \quad \text { Eqn } 4
$$

The non-dimensional force-deflection equations for bodies of different sizes, materials and magnitude of loading are identical to each other provided that the value of the bracketed quantity is preserved. Using the subscripts A to denote parameters pertinent to the airplane and $\mathrm{M}$ to denote parameters pertinent to the wind tunnel model, the requirement for force-deflection similitude is given by

$$
\left(\frac{\bar{q} L^{4}}{E_{0} I_{0}}\right)_{A}=\left(\frac{\bar{q} L^{4}}{E_{0} I_{0}}\right)_{M}
$$

which can be rewritten as

$$
\frac{\left(E_{0} I_{0}\right)_{M}}{\left(E_{0} I_{0}\right)_{A}}=\frac{\bar{q}_{M}}{\bar{q}_{A}}\left(\frac{L_{M}}{L_{A}}\right)^{4}
$$

Defining scale factors, $\lambda$, as the ratios of wind tunnel model parameters to airplane parameters, eqn 6 can be written

$$
\lambda_{E I}=\lambda_{\bar{q}} \lambda_{L}^{4}
$$

Equationss 4 through 7 each mathematically express that deflection similitude requires equal values for the ratio of applied force to structural stiffness. For the static aeroelastic problem, the applied force is an aerodynamic load. Maintaining the relationship in eqn 7 is essential for static aeroelastic scaling.

Freedoms, Constraints \& Choices
The previous discussions lead to six free parameters or independent variables in the cantilevered static aeroelastic model scaling problem: Mach number of the aircraft, altitude of the aircraft, Mach number of the model, dynamic pressure of the model, size and stiffness of the model. Additional variables are neglected, such as Reynolds' number and mass ratio; others are fixed, such as size and stiffness of the flight vehicle.

Two constraints are applied, as dictated by the similitude requirements previously discussed: Mach number must be matched and the ratio of stiffness to aerodynamic force must be matched. This reduces the number of free parameters to four. There are two important aspects implicit in the force-deflection relationship development: the distribution of the stiffness must be maintained and the distribution of the aerodynamic forces must also be maintained ${ }^{5}$.

Design of the wind tunnel model requires that choices be made. A length scale factor and a stiffness scale factor must be selected, thereby fixing model size and stiffness, and reducing the number of free parameters to two.

These two remaining independent variables are chosen during the planning and conducting of the tests. During testing, there are four possible freedoms: Mach number of the airplane, altitude of the airplane, Mach number of the wind tunnel and dynamic pressure of the wind tunnel. Only two of these are still independent variables. Choice of airplane Mach number and altitude, exercised as choices of the two remaining free parameters, specifies a corresponding wind tunnel test Mach number and dynamic pressure.

It is important to note that the problem is not over constrained. For every choice of Mach number and altitude of the airplane, there will be a corresponding Mach number and dynamic pressure in the wind tunnel. Thus, all flight test conditions can be mapped to wind tunnel test conditions.

\section{WAM Methodology}

Application of the WAM methodology involves several steps. The scale factors are developed based on a single flight condition in the vehicle's flight envelope and a single test condition in the wind tunnel. These choices of test conditions must take into consideration the requirements for applying the scale factors throughout the test envelopes; these considerations will be discussed shortly. The wind tunnel test points that correspond to the remaining points in the flight test envelope are produced using the scale factors determined from that initial point. Corrections to the wind tunnel test conditions or to the flight test conditions can be made after the first data set (either flight data or wind tunnel data) is 
acquired. These corrections are discussed in a subsequent section.

\section{Developing Scale Factors}

The four principal scale factors for static aeroelastic scaling have been shown to be Mach number, length, structural stiffness, dynamic pressure and Mach number. The Mach number scale factor must be 1 for aerodynamic similitude and the other three must be related as shown in eqn 7 . The designer of a static aeroelastically scaled wind tunnel model has freedom to assign values to two of these three scale factors. The third becomes a dependent variable in the design process. Historically and for practical reasons, the scale factor on stiffness has been relegated to be the dependent variable.

The two choices to be made then are the scale factors for dynamic pressure and length. The dynamic pressure scale factor can be determined by choosing an altitude and Mach number of the airplane and a wind tunnel dynamic pressure to correlate at that condition. The geometric length scale factor can either be selected based on wind tunnel test section dimensions, to match the Froude numbers or to meet some other test criteria. Once these decisions have been made, the constraints requiring Mach number matching and ratio of stiffness to aerodynamic force matching, eqn 7 , fix the remaining scale factors.

\section{Considerations for Multi-point Scaling}

There are several practical issues of wind tunnel model construction and testing to bear in mind when developing the scale factors. The choice of scale factors must comply with wind tunnel test section size and operational capabilities.

The goal of multi-point scaling is to have the entire flight envelope map into the wind tunnel operating envelope. Factors that limit the capability of a wind tunnel include fan speed, blockage, contraction ratio and inefficiencies. As an example, at its maximum Mach number of 1.2 the TDT has a dynamic pressure limit of $340 \mathrm{psf}$ when operating in heavy gas. For lower Mach numbers, the dynamic pressure capabilities are higher. It is recommended that a high value of the wind tunnel dynamic pressure at the maximum Mach number to be flown be used to determine the stiffness scale factor.

Using a lower dynamic pressure for scaling has repercussions on the model design. Lowering the maximum scaling dynamic pressure lowers the aerodynamic force applied to the model. Recall that the ratio of stiffness to aerodynamic force is a parameter that must be scaled properly between the model and the aircraft. Thus, as the dynamic pressure for testing the model is reduced, the stiffness of the model must also be reduced. Finding material that is sufficiently strong, but not overly stiff, is usually the crux of the design problem. The loads applied have been reduced, so the material doesn't have to be as strong, but it also can't be as stiff. This chasing of material properties relative to applied loads and model safety criteria is a significant challenge in model design.

It is desired to map the maximum Mach number at the minimum flight test altitude to a high dynamic pressure in the wind tunnel. The designer should consider leaving some margin in dynamic pressure between the maximum scaled wind tunnel test condition and the wind tunnel operating limits because 1) the conditions being flown will not always be precise; 2) the conditions being flown may be changed at some point in the test planning; 3 ) the atmosphere isn't usually at standard-day conditions; 4) the speed of sound in the wind tunnel is a function of gas purity and temperature, neither one of which is within the experimenter's control, i.e. these quantities are not known exactly ahead of time; and 5) placing a model in the tunnel changes the maximum operating conditions of the tunnel. This latter point is particularly important if a splitter plate or a large model is being considered.

\section{Determining wind tunnel test points}

For the chosen scale factors, a single wind tunnel model is built, cementing the length and stiffness scale factors. The ratio of the dynamic pressures of the flight test conditions and the wind tunnel test conditions is thus also cemented due to the constraint given by eqn 7 . Combining eqn 7 with the definition of the dynamic pressure scale factor and definitions of Mach number and dynamic pressure yields the following requirement on wind tunnel dynamic pressure.

$$
\begin{array}{cc}
\overline{\boldsymbol{q}}_{\boldsymbol{M}}=\frac{\lambda_{E I}}{\lambda_{L}^{4}} \overline{\boldsymbol{q}}_{A} & \text { Eqn } 8 \\
\overline{\boldsymbol{q}}_{A}=\mathbf{1} / \mathbf{2}\left(\rho \boldsymbol{a}^{\mathbf{2}}\right)_{A} \boldsymbol{M}_{A}^{2} & \text { Eqn } 9 \\
\overline{\boldsymbol{q}}_{\boldsymbol{M}}=\mathbf{1} / \mathbf{2}\left(\frac{\lambda_{E I}}{\lambda_{L}^{4}}\right)\left(\rho a^{2}\right)_{A}\left(\boldsymbol{M}^{2}\right)_{A} & \text { Eqn } 10
\end{array}
$$

The terms have been grouped to emphasize the connections with the airplane parameters. Once created or built, the wind tunnel model has a certain size and stiffness, which are related to the full-scale quantities by the length scale factor and the stiffness scale factor, fixing the first term in parentheses. The second parenthesized term is a function of aircraft altitude; the third is a 
function of aircraft Mach number. The wind tunnel must have the capability to achieve a range of dynamic pressure-Mach number combinations to satisfy eqn 10 for all altitude-Mach number combinations to be flight tested.

As suggested by eqn 10, the test parameters- Mach number, aircraft altitude and wind tunnel dynamic pressure- form a three-dimensional space. Within this three-dimensional test space, a surface exists that corresponds to a single static aeroelastically scaled wind tunnel model -flight vehicle pair. The shape of the surface depends on the values of the length and stiffness scale factors. The surfaces are referred to as WAM surfaces. Figure 2 shows three possible WAM surfaces in the test space, corresponding to three different values of the stiffness scale factor. The length scale factor has been held constant. Figure 3 shows three different possible surfaces in the test space, corresponding to three values of length scale factor. The stiffness scaling has been kept constant in producing these surfaces.

A single WAM surface, as illustrated in Figure 4, is produced by choice of length and stiffness scale factors; the various points on the surface define flight test point/wind tunnel test point pairs. An example test point pair is denoted by the black circle lying on the surface at Mach 1.2, flight altitude $5000 \mathrm{ft}$ and wind tunnel dynamic pressure 369 psf. The projection of this pair onto the flight test surface and the wind tunnel test surface are shown in the figure by the blue circles. Figure 5 shows the projection of the entire WAM surface from figure 4 onto the flight test plane. All combinations of Mach number from 0.85 to 1.2 and altitude from 5000 to $30000 \mathrm{ft}$ are considered as possible flight test points. Figure 6 shows the projection of the WAM surface from figure 4 onto the wind tunnel test plane. The corresponding wind tunnel test points occupy the colored region from Mach 0.85 to 1.2 and dynamic pressure from 66 to $369 \mathrm{psf}$.

In comparing the flight test envelope and the wind tunnel test envelope, it is important to recognize that the highest altitude point at a given Mach number is mapping into the lowest dynamic pressure point. It is interesting to note that a line of constant flight vehicle altitude maps very nearly to a line of constant wind tunnel density.

Requiring a match of the Froude number throughout the test space would lead to a single line of scaled points instead of a surface. This line would correspond to a line of constant flight vehicle altitude and a line of roughly constant wind tunnel density.

\section{Hypothetical Wing}

A simple testcase was generated to demonstrate the application of the WAM methodology. Two analytical models were generated: one representing the wing of a flight vehicle and one representing a wind tunnel model that is static aeroelastically scaled to the flight vehicle. Static aeroelastic analysis was performed at Mach 0.85 over a range of dynamic pressures, producing stability and control derivatives for both analytical models.

The models consisted of pinned-root wings, each with an aileron. An approximate planform of an F-18 wing provided the geometry for this testcase. In this simple example, length and dynamic pressure scale factors were selected without regard to scaling to actual test points, testing within the limitations of a given facility or using actual materials. The wind tunnel model was geometrically scaled to be $1 / 4$ the size of the flight vehicle scale wing. The dynamic pressure scale factor was specified as $1 / 5$.

Substituting these values into eqn 7 gives the stiffness scale factor, eqn 11.

$$
\lambda_{E I}=\lambda_{q} \lambda_{L}^{4}=0.2 \times 0.25^{4}=0.00078125
$$

In this example, the material thickness was scaled using the length scale factor, establishing the scale factor on area moment of inertia, eqn 12. The material stiffness (Young's modulus) was scaled such that eqn 7 was obeyed, eqn 13.

$$
\begin{array}{cc}
\lambda_{E I}=\lambda_{E} \lambda_{I}=\lambda_{E} \lambda_{L}^{4} & \text { Eqn } 12 \\
\lambda_{E I}=\lambda_{q} \lambda_{L}^{4}=\lambda_{E} \lambda_{L}^{4} \longrightarrow \lambda_{q}=\lambda_{E}=\mathbf{0 . 2} & \text { Eqn } 13
\end{array}
$$

Note that the same effect could be produced by maintaining the same material for the flight vehicle and wind tunnel model, but scaling the material thickness separately from the in-plane geometry. A combination of these two approaches is more like the procedure required in designing an actual wind tunnel model.

The structure of the main wing was a flat plate with a thickened aft-sweeping region. The aileron was a single-thickness plate, structurally connected to the wing by two tabs. The structural finite element model with element thickness is shown in the figure 7. The thicknesses in the figure are for the wind tunnel model scale; due to the length scale factor chosen, thickensses for the flight vehicle are 4 times as large.

Static aeroelastic trim analyses were run for these models. The resulting rigid pressure distributions, aeroelastic pressure distributions and deflections were compared. The results were identical in pattern and correctly scaled relative to each other.

The lift curve slope, $\mathrm{C}_{\mathrm{L \alpha}}$, and the lift coefficient due to aileron deflection, $\mathrm{C}_{\mathrm{L} \delta}$, are shown in figures 8 
and 9. In comparing the results, the dynamic pressures associated with the wind tunnel model were multiplied by the dynamic pressure scale factor that was generated in association with the model scaling. The results are identical, demonstrating that for a simple construction flight vehicle wing, using hypothetical materials without any strength consideration, without any rigid body motion influences, without any wind tunnel effects, or other complicating real-world effects, the WAM scaling and comparison methodology work perfectly.

\section{Active Aeroelastic Wing}

The following shows an example of applying the WAM methodology to the scaling of the Active Aeroelatic Wing (AAW) wind tunnel model. In this actual design case, the flight test points had been established and the capabilities of the TDT were employed in determining the various scale factors and wind tunnel test points. Because this model is actually being built, it is required that it be constructed of materials which actually exist, rather than just as mathematical constructs.

This application of the WAM methodology proceeds in two steps: 1) selection of the test point pair, which fixes the dynamic pressure scale factor and 2) selection of the length scale, which in combination with step 1 and eqn 7 fixes the stiffness scale factor. These two steps can also be described as choosing a point in the 3-dimensional test space and then choosing from the infinite number of WAM surfaces that pass through that point. The details of these choices follow.

A Mach number and altitude combination was chosen from the flight test envelope. Choosing an altitude fixed the speed of sound and the density to be used in the scaling process. For developing the scale factors, a standard atmosphere was assumed; implications of this assumption are discussed later. Through these choices, the flight vehicle's dynamic pressure and velocity were specified.

Chosen flight test values:

$$
\mathrm{M}_{\mathrm{A}}=1.2 \quad \mathrm{~h}_{\mathrm{A}}=15,000 \mathrm{ft}
$$

Resulting flight condition parameters:

$\mathrm{a}_{\mathrm{A}}=1057 \mathrm{ft} / \mathrm{sec}$

$\rho_{\mathrm{A}}=0.001496$ slugs $/ \mathrm{ft} 3$

$\overline{\boldsymbol{q}}_{\boldsymbol{A}}=1 / 2 * \rho_{\mathrm{A}} * \mathrm{M}_{\mathrm{A}}{ }^{2} * \mathrm{a}_{\mathrm{A}}{ }^{2}=1204 \mathrm{psf}$

$\mathrm{V}_{\mathrm{A}}=\mathrm{M}_{\mathrm{A}} * \mathrm{a}_{\mathrm{A}}=1269 \mathrm{ft} / \mathrm{sec}$

A dynamic pressure was chosen for the wind tunnel test condition to correspond to the above flight test condition. This choice established the dynamic pressure scale factor. In the scaling process, estimated gas purity and operating total temperature have to be assumed. Based on historical TDT data, the values chosen for use were $95 \%$ purity and $100^{\circ} \mathrm{F}$. These are estimates of the conditions that will occur in the tunnel, not parameters over which control can be exercised. The consequences of errors in these estimates will be discussed subsequently.
The Mach number must be the same as the flight vehicle. Specifying Mach number, dynamic pressure, purity and temperature establishes the other variables of the wind tunnel, such as velocity, speed of sound, density, and pressure. Thus, scale factors for speeds of sound, velocity, and density have also been established.

Chosen wind tunnel test values:

$$
\mathrm{M}_{\mathrm{M}}=1.2 \quad \overline{\boldsymbol{q}}_{\boldsymbol{M}}=250 . \mathrm{psf}
$$

Resulting wind tunnel parameters:

$\mathrm{a}_{\mathrm{M}}=540 . \mathrm{ft} / \mathrm{sec}$

$\mathrm{V}_{\mathrm{M}}=\mathrm{M}^{*} \mathrm{a}_{\mathrm{M}}=648 \mathrm{ft} / \mathrm{sec}$

$$
\rho_{\mathrm{M}}=2 * \mathrm{q}_{\mathrm{M}} / \mathrm{V}_{\mathrm{M}}{ }^{2}=0.0011907 \text { slugs } / \mathrm{ft}^{3}
$$

As a result of choosing the above test point pair, the dynamic pressure scale factor is fixed, eqn 14.

$$
\lambda_{\bar{q}}=\frac{\bar{q}_{M}}{\bar{q}_{A}}=\left(\frac{250 p s f}{1204 p s f}\right)=\mathbf{0 . 2 0 7 6} \quad \text { Eqn } 14
$$

The second major step in applying the WAM procedure for this application is choosing the length scale factor. The length scale factor was chosen through the Froude number, eqn 15. The choice was made to match the Froude number, eqns 16 and 17, at the test point pair designated previously. The model will not be Froude scaled for other points in the test envelopes. As discussed previously, Froude scaling is not required. It produced a convenient size scale factor in this instance, eqn 18.

$$
\begin{array}{cc}
\boldsymbol{F r}=\frac{\boldsymbol{V}}{\sqrt{\boldsymbol{g b}}} & \text { Eqn 15 } \\
\boldsymbol{F r}_{\boldsymbol{A}}=\boldsymbol{F} \boldsymbol{r}_{\boldsymbol{M}} & \text { Eqn 16 } \\
\frac{\boldsymbol{b}_{\boldsymbol{M}}}{\boldsymbol{b}_{\boldsymbol{A}}}=\left(\frac{\boldsymbol{V}_{\boldsymbol{M}}}{\boldsymbol{V}_{\boldsymbol{A}}}\right)^{\mathbf{2}} & \text { Eqn 17 } \\
\lambda_{\boldsymbol{L}}=\lambda_{V}^{\mathbf{2}}=\left(\frac{\mathbf{6 4 8}(\boldsymbol{f t} / \mathrm{sec})}{\mathbf{1 2 6 9}(\boldsymbol{f t} / \mathbf{s e c})}\right)^{\mathbf{2}}=\mathbf{0 . 2 6 0 9} & \text { Eqn } 18
\end{array}
$$

The stiffness scale factor is determined, eqn 19, by enforcing eqn 7 . Figures 4 through 6 show the WAM surfaces corresponding to this example.

$$
\lambda_{E I}=\lambda_{q} \lambda_{L}^{4}=0.2076 \times 0.2609^{4}=\mathbf{0 . 0 0 0 9 6 2} \quad \text { Eqn } 19
$$

Using these scale factors, finite element models of the flight vehicle and the wind tunnel model design have been developed and analyzed. The aerodynamic models utilized in this study are linear models; the wind tunnel model's aerodynamic box layout is a geometrically scaled version of the flight vehicle's aerodynamic box layout. Subsonic analyses utilize doublet lattice aerodynamic theory; the supersonic analyses use ZONA51 aerodynamics.

The AAW wind tunnel model is structurally composed of a contoured center plate that will have a balsa wood aerodynamic shell applied to it. The contouring of the center plate was the result of an iterative design process, matching first the structural 
stiffness properties and then the aeroelastic properties of the flight vehicle. The resultant conceptual design for the center plate is shown in figure 10.

Static aeroelastic analyses were performed; stability and control derivatives were calculated for the configurations assuming first that the flight vehicle and wind tunnel model are rigid and second with flexibility incorporated. Sample analytical results are shown in figures 11 through 13. As with the simple test case, the rigid aerodynamic results were identical for the flight vehicle and the wind tunnel model. Figure 11 shows the test conditions for the flight vehicle at $5000 \mathrm{ft}$ and the wind tunnel model at corresponding wind tunnel test conditions. These test conditions correspond to test points shown on the WAM surface in figures 4 through 6 by the dashed green trajectory lines.

Figures 12 and 13 show the change in control surface effectiveness for the leading edge outboard control surface and the aileron. Both are presented as functions of Mach number. Four sets of data are plotted in each figure: the flight vehicle assuming no flexibility (rigid); the flight vehicle with aeroelastic effects at $5000 \mathrm{ft}$ altitude; the wind tunnel model assuming no flexibility (rigid); and the wind tunnel model with aeroelastic effects at the wind tunnel dynamic pressures corresponding to $5000 \mathrm{ft}$. The trends shown in the figures correspond to expected trends for an aft-swept wing. The trailing edge control surface is less effective with flexibility incorporated. The leading edge control surface becomes more effective with flexibility effects incorporated.

Figure 12 shows that the values of $\mathrm{C}_{\mathrm{L} \delta \mathrm{LEO}}$ for the rigid flight vehicle and the rigid wind tunnel model are identical, showing that the scaling of the aerodynamic loads and the geometry are correct. Comparing the aeroelastic coefficients to the rigid coefficients shows that in both the flight vehicle and the wind tunnel model design, there is a substantial change in the coefficient. Both the flight vehicle and wind tunnel model exhibit the same trends with Mach number and with incorporation of the aeroelastic effects. However, the change in the leading edge outboard control surface effectiveness is larger for the flight vehicle than for the wind tunnel model in the transonic regime, indicating that some areas of the wind tunnel model are stiffer than those areas of the flight vehicle. The root cause of this difference is associated with fabrication and testing constraints. Testing requirements in NASA Langley wind tunnels force the model design to be very strong, requiring that certain parts of the wing cannot be made as thin as would be required for matching the properties of the flight vehicle more precisely. This mis-match in properties is particularly true for regions near the leading edge control surfaces, where substantial material thickness had to be added to meet strength requirements.

In figure 13 the values of $C_{L \delta a i l}$ for the rigid flight vehicle and the rigid wind tunnel model are identical.
For both the flight vehicle and the wind tunnel model design, the coefficient changes substantially with the incorporation of flexibility. In the case of the aileron effectiveness, both the trend and the magnitude agree well in comparing the wind tunnel model and the flight vehicle. Because the hinge moments being applied to and by the trailing edge control surface are much lower than those encountered by the leading edge control surface, the strength requirements are not nearly as restrictive. The wind tunnel model designer had sufficient freedom to tailor the material thickness distribution near the aileron and in its load conduction path. Thus, the control derivative associated with the aileron matches that of the flight vehicle more closely than that associated with the leading edge control surface derivative.

Because this is an example of an actual design process, with the required design compromises for construction and testing, the comparisons of static aeroelastic properties are not as good as demonstrated in the previous simple scaling example.

\section{Influence of changes, errors, and uncertainties}

Many issues that can arise as data is acquired influence the WAM process and data interpretation. Additionally, known issues exist which will limit precision of test point correlation. These issues and their anticipated influences are presented.

\section{Accounting for pilot being off-condition or} non-standard atmospheric conditions

Small variations in the aircraft altitude or Mach number can be accounted for by altering the wind tunnel test conditions, in effect moving to a different point on the WAM surface. In altering the wind tunnel test point that is to correspond to the new flight test condition, the Mach number must match the actual flight test Mach number. The dynamic pressure for wind tunnel test point should be calculated using the actual dynamic pressure obtained during the flight test.

To account for a non-standard day, the corresponding mapped surface can be generated by adjusting the table of densities and speeds of sound corresponding to a given altitude. A different altitude or velocity means that the wind tunnel's matching condition is a different point on the original mapped surface.

\footnotetext{
Effect of wind tunnel speed of sound variations

The speed of sound in the TDT is primarily a function of the purity and temperature of the gas. When R134a was first introduced at the TDT, a speed of sound of $525 \mathrm{ft} / \mathrm{sec}$ was predicted. When
} 
experimental data was obtained, a value of $540 \mathrm{ft} / \mathrm{sec}$ was measured. The difference is primarily attributed to the purity of the gas being less than $100 \%$, i.e. the test medium is a mixture of air and R-134a. Since the speed of sound in air $(1116 \mathrm{ft} / \mathrm{sec})$ is substantially higher than the speed of sound in heavy gas, even a $1 \%$ impurity raises the test medium speed of sound noticeably.

The Froude number of the airplane and the model match when the ratio of their speed of sound squared equals the length scale factor.

$$
\lambda_{\mathrm{L}}=\mathrm{a}_{\mathrm{M}}{ }^{2} / \mathrm{a}_{\mathrm{A}}{ }^{2}
$$

If the speed of sound in the wind tunnel differs from that used to originally compute the length scale factor, the model is now Froude scaled for a different airplane speed of sound and therefore different altitude.

The changed speed of sound affects intermediate scale factors for velocity and density, but in such a way that their individual effects cancel each other when computing the stiffness scale factor. The same is true for the dynamic pressure point. The dynamic pressure at which a wind tunnel test point is acquired is not changed because of an inaccuracy in the tunnel speed of sound employed in the design procedure. Preserving the dynamic pressure, however, means that the density and speed of sound differ from those used in the design process. The density and speed of sound of the tunnel test medium will be different for different purities. This is an issue of practical concern, as the tunnel has leaks, which result in a decrease in the gas purity. The observable effect is a reduction in tunnel operating limit.

Measurement uncertainties and instrument resolution limitations

Uncertainties in wind tunnel measurements and resolution limitations of instruments, herein referred to as measurement errors, were the subjects of a parameter sensitivity study. The results provide limits for how precisely a specified flight test condition can be matched using the wind tunnel instrumentation. Conversely, the results provide bounds for the precision with which the flight test condition should be specified for purposes of correlating with the wind tunnel results. To perform this study, flight test points were mapped into the TDT test space using the WAM methodology and expressed in terms of primary tunnel parameters. These calculated parameters serve as simulated measurements, to which measurement errors were applied. Tunnel parameters reflecting these measurement errors were computed and mapped back to the flight test Mach number and altitude. Applying this procedure required the dynamic pressure scale factor, standard atmosphere tables, and the TDT tunnel parameters program. $^{2}$

Instruments that measure total pressure, static pressure, gas purity and total temperature specify the test conditions in the TDT. These measurements are made and values input to a tunnel parameters computer program that computes additional flow parameters, including Mach number and dynamic pressure.

For this study, the resolutions of the pressure readings were assumed to be $0.1 \mathrm{psf}$, the purity of the gas $0.1 \%$ and the total temperature $0.1^{\circ} \mathrm{F}$. Additionally, the uncertainties have been established as follows. For the pressures, the manufacturer of the transducer quotes the accuracy as $0.016 \%$ of reading $+/-0.008 \%$ of full scale (full scale reading $2100 \mathrm{psf}$ ). A temperature fluctuation of $10^{\circ} \mathrm{R}$ was used in this study. The gas purity measurement is assumed accurate to $+/-3 \%$.

The influence of measurement errors was examined at the maximum and minimum test conditions flown in the AAW flight test program. The results for the Mach 0.85, $5000 \mathrm{ft}$ flight test point are presented in figure 14, which represents a very small region of the projection of the WAM surface, figure 4 , onto the flight test plane. The conclusions, summarized below, indicate the impact of measurement errors on test condition specifications and also indicate which measurement errors most significantly impact the ability to precisely match flight test conditions.

Instrument resolution used in measuring TDT tunnel parameters translates into flight test altitude resolution of 31 feet. This is the additive resolution associated with the instruments used in measuring total pressure, static pressure, temperature and heavy gas purity. Instrument resolution also impacts Mach number resolution. Mach number can be resolved to 0.0015 .

Expanded uncertainties of measurements were also examined. Uncertainty in the total pressure primarily impacts the Mach number. Uncertainty in static pressure significantly affects both Mach number and altitude (110 ft). The temperature uncertainty produces Mach number uncertainty $(0.001)$ and altitude uncertainty $(65 \mathrm{ft})$. The purity uncertainty produces Mach number uncertainty of 0.002 and altitude uncertainty of $132 \mathrm{ft}$.

Variability in TDT temperature and purity can cause misinterpretation of mis-scaling of altitude. To avoid the mis-scaling, scale each flight condition using the dynamic pressure scale factor and use realtime measurements of tunnel temperature and purity. 


\section{Concluding Remarks}

Static aeroelastic wind-tunnel research being performed at NASA Langley Research Center is an essential part of the Active Aeroelastic Wing (AAW) program. Wind Tunnel to Atmospheric Mapping (WAM) is the process through which the wind-tunnel model is scaled to correlate with the AAW Flight Vehicle. WAM involves adapting the classic aeroelastic scaling laws for developing statically scaled wind tunnel models, circumventing some of the difficult issues associated with typical aeroelastically scaled models. For this type of testing, a practical wind tunnel model can be designed and built such that multiple flight vehicle test conditions map to the wind tunnel envelope. For the AAW and similar static aeroelasticity programs, matching multiple test conditions would not be possible if the traditional scaling requirements used on flutter models were imposed, specifically mass ratio, reduced frequency and Froude number matching. Characteristics of the NASA Langley Transonic Dynamics Tunnel, particularly the variable pressure capability and the heavy-gas test medium, enable the multi-point scaling.

Reproducing the static aeroelastic characteristics of a full-scale vehicle in a wind tunnel model requires that several non-dimensional parameters be identical for both vehicle and model. Similitude requires that testing be conducted at matching Mach numbers and that the ratio of stiffness to aerodynamic forces must be the same. The aerodynamic pressure distribution and the stiffness distribution within the structure must also be maintained. In the static aeroelastic design and test space, two free parameters remain after the model is constructed. These freedoms consist of the Mach number and either the altitude of the flight vehicle or the Mach number and dynamic pressure of the wind tunnel model. Variation of the free parameters and calculation of the third creates a 3-dimensional surface on which the wind tunnel model and the flight vehicle operate. Thus, all flight test conditions map to wind tunnel test conditions.

Detailed design of a model to duplicate the static aeroelastic response of a flight test vehicle requires much diligence and balancing of strength criteria with stiffness matching requirements, as discussed in the AAW design problem. Model safety criteria dictating strength requirements cut into the ability to produce a model that is a precise representation of the flight vehicle.

\section{References}

1 Molyneux, W. G. , “Aeroelastic modeling”, RAE Technical note number Structures 353, March 1964.
2 Kvaternik, R.G., "Computer programs for calculating the isentropic flow properties for mixtures of R-134a and air," NASA TM-2000210622, November 2000.

3 Keller, D.F., Farmer, M., Kvaternik, R.G., and Cole, S.R., Personal communications, scaling computer program and unpublished documents including (Kvaternik, R.G. "Computer Programs for Calculating the Isentropic Flow Properties for Mixtures of R-134a and air: Source code listings," November 2000)

4 Kuntz, W.H., Wasserman, L.S. and Alexander, H.R., "Dynamically similar model tests of rotary wing and prop types of VTOL aircraft"

5 Bisplinghoff, Ashley and Halfman, "Aeroelasticity," Addison-Wesley Publishing, Reading, Massachusetts, 1955.

6 Guyett, P.R., "The use of flexible models in aerospace engineering," RAE Technical Report No. 66335, November 1966.

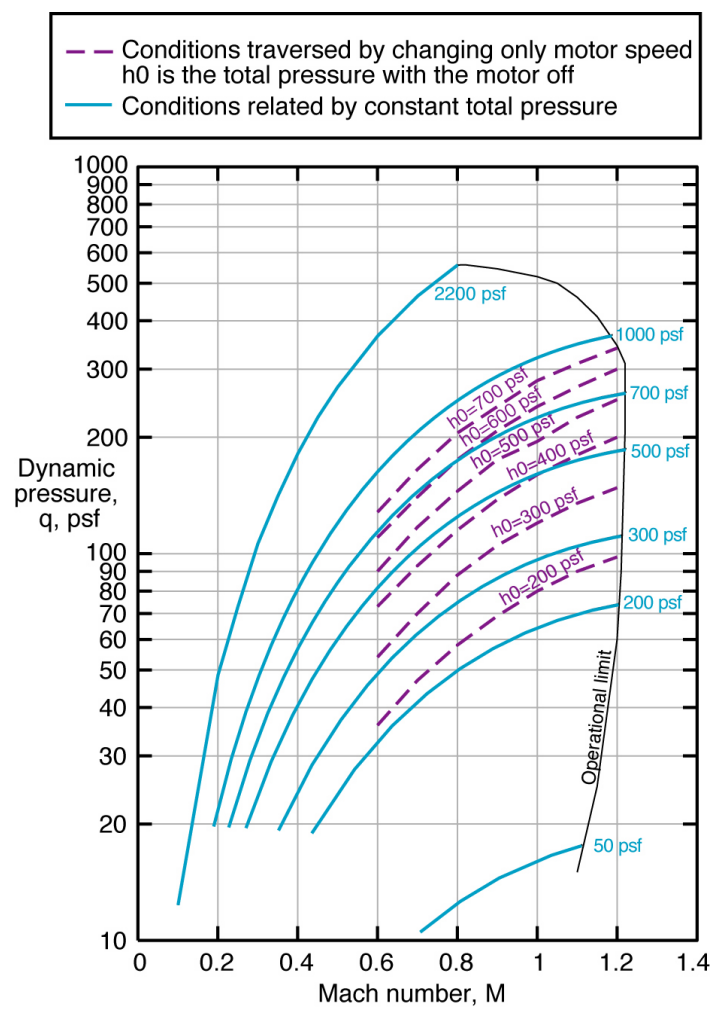

Figure 1. Test envelope of the Transonic Dynamics Tunnel, operating with heavy gas test medium 


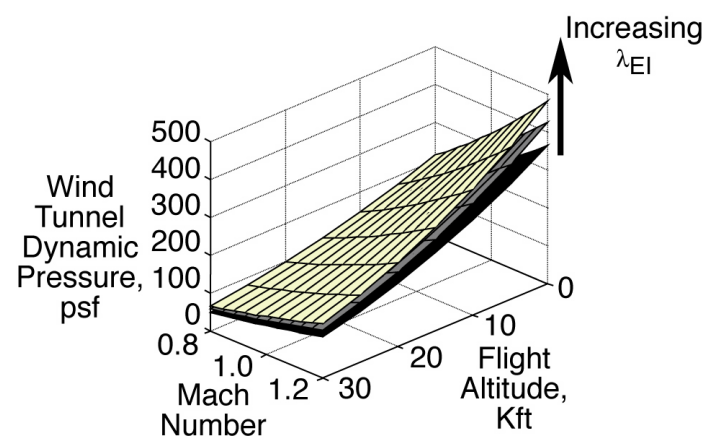

Figure 2. WAM surfaces for several stiffness scale factors; length scale factor $=0.26$

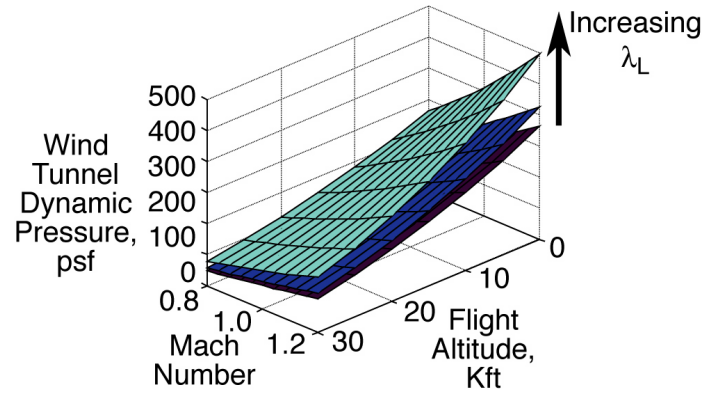

Figure 3. WAM surfaces for several length scale factors; stiffness scale factor $=0.00962$

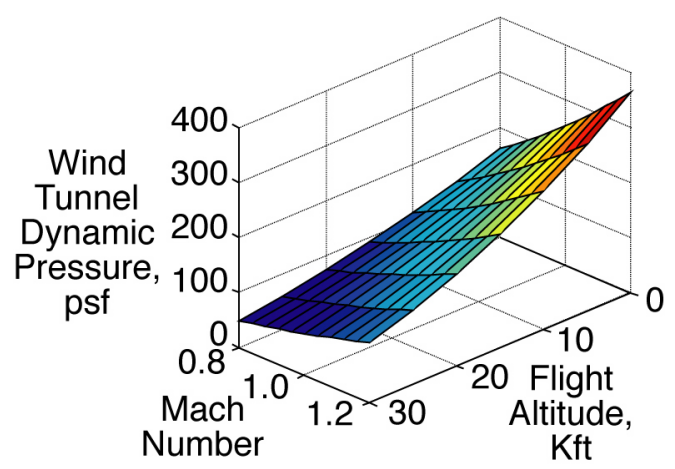

Figure 4. WAM surface for stiffness scale factor $=$ 0.00962 , length scale factor $=0.26$

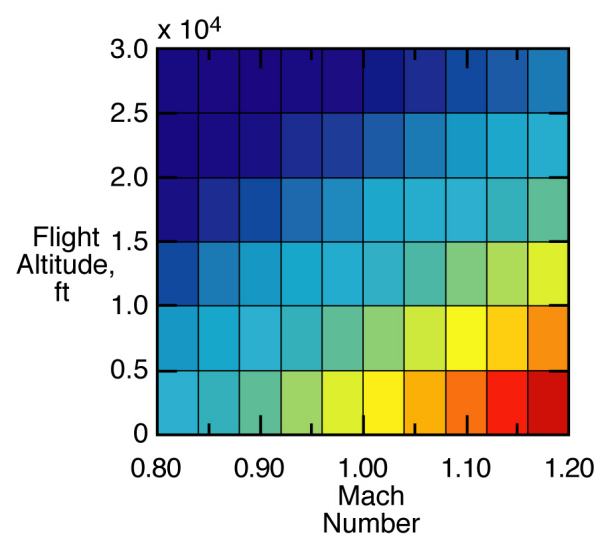

Figure 5. WAM surface showing flight vehicle test envelope

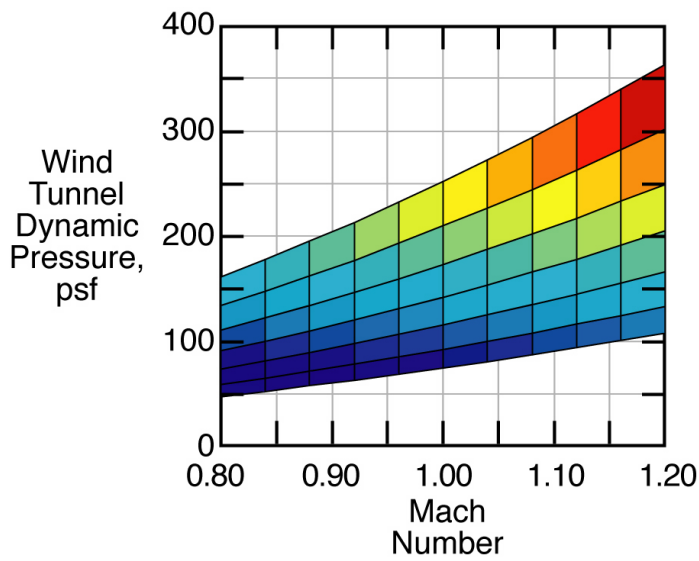

Figure 6. WAM surface showing wind tunnel test envelope

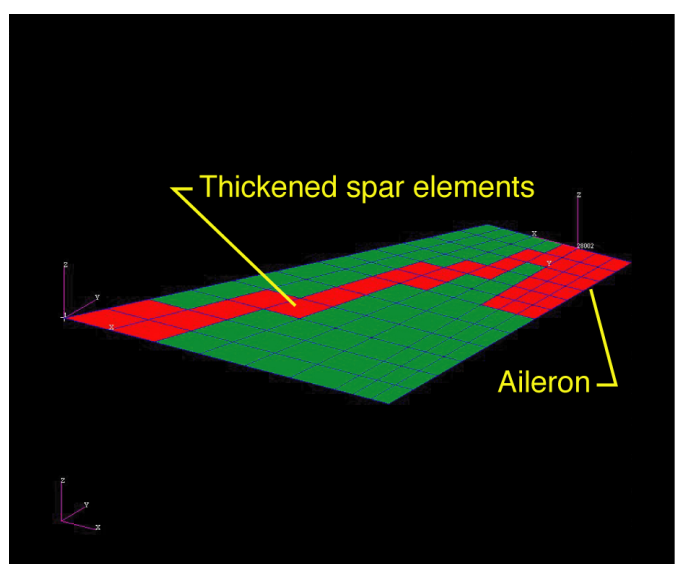

Figure 7. Structural model of hypothetical wing test case

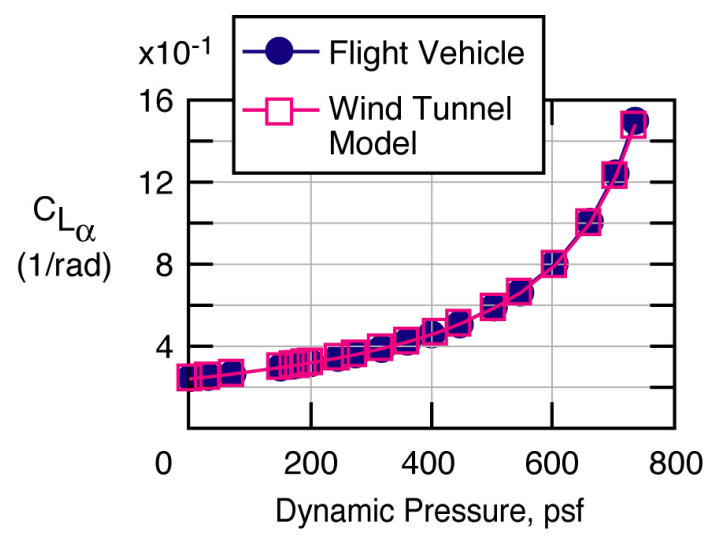

Figure 8. Comparison of hypothetical wing flight vehicle and wind tunnel model: lift curve slope 


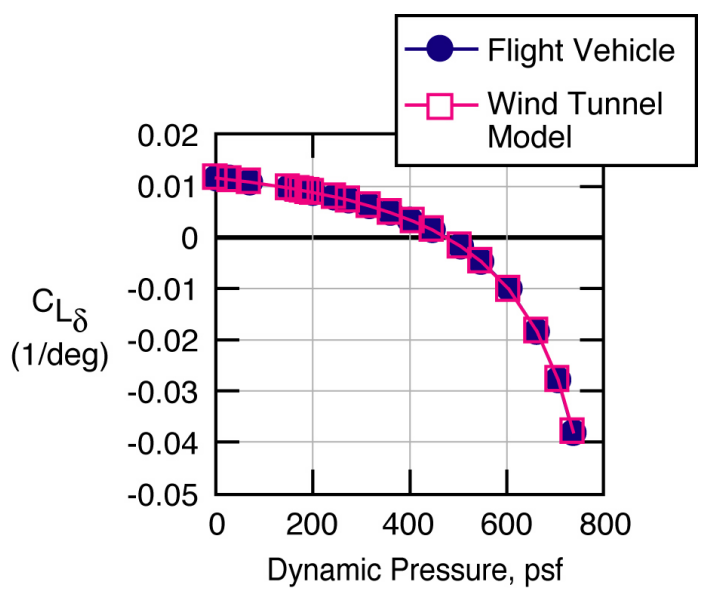

Figure 9. Comparison of hypothetical wing flight vehicle and wind tunnel model: lift coefficient due to control surface deflection

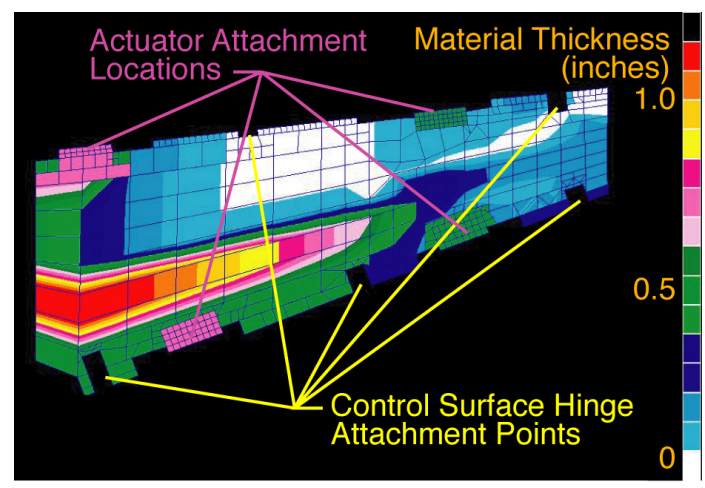

Figure 10. Conceptual design of wing core for AAW wind tunnel model; planform view showing material thickness

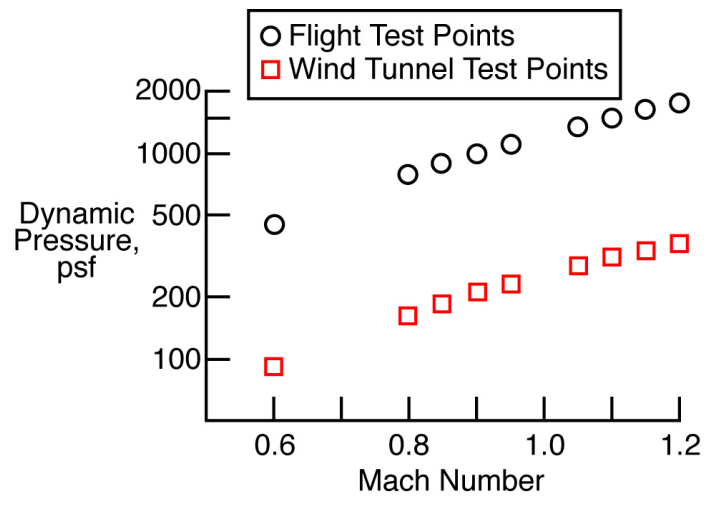

Figure 11. Test points corresponding to $5000 \mathrm{ft}$ altitude

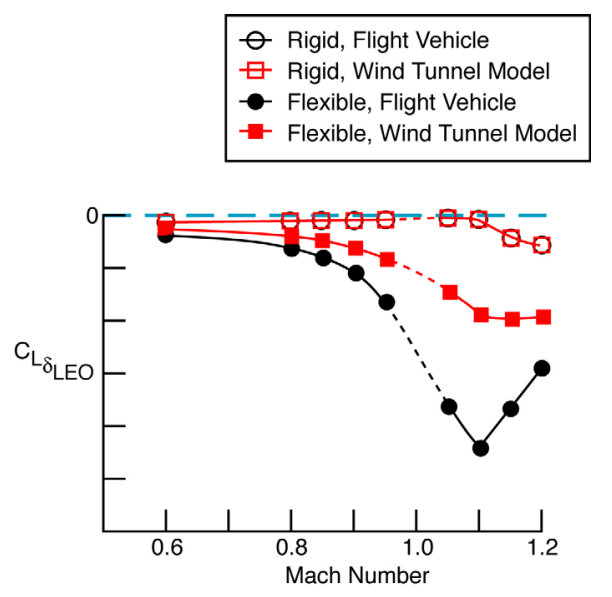

Figure 12. Comparison of AAW analytical results for flight vehicle and wind tunnel model; lift coefficient due to leading edge outboard control surface deflection

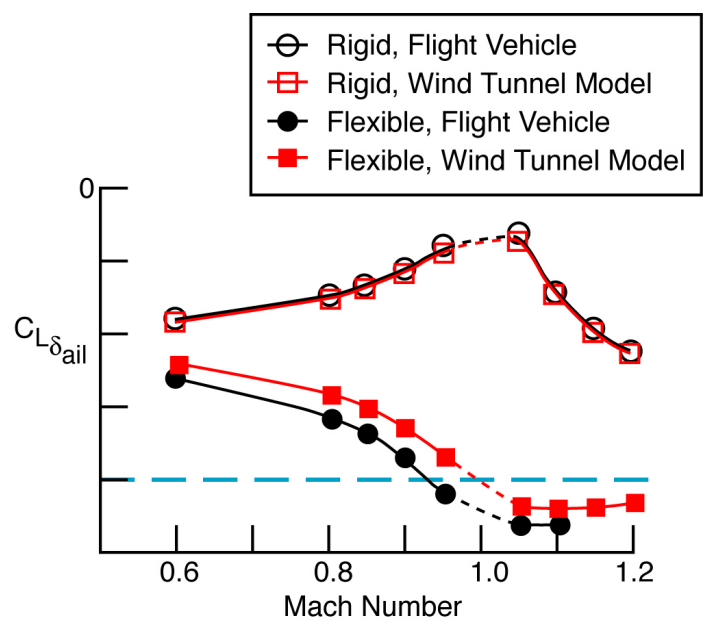

Figure 13. Comparison of AAW analytical results for flight vehicle and wind tunnel model; lift coefficient due to trailing edge outboard control surface (aileron) deflection

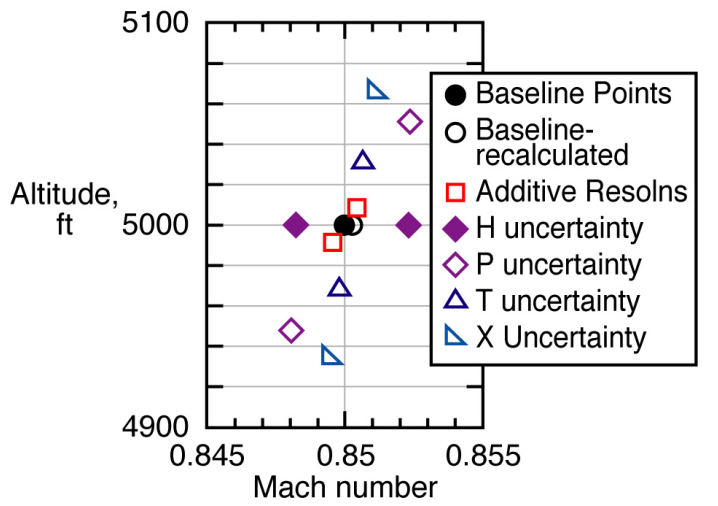

Figure 14. Effect of wind tunnel measurement uncertainties and resolution limitations for the flight test point at Mach $0.85,5000 \mathrm{ft}$ altitude 